overlong in adverse conditions. In cold weather the blood should be kept in an incubator at blood heat for a quarter of an hour before the test is applied. Excepting for this the shorter the interval between collecting the blood and its being used the better. If the corpuscles are left very wet they should be evenly distributed by a circular twist of the tube. Indeed, this is in any case a wise precaution. Too rapid centrifuging is stated to cause clumping of the phagocytes and to lower the index.

In preparing the bacterial suspension a relatively strong salt solution (1.6 per cent.) should be used, as Wright has shown that weak saline solution will excite some phagocytosis even without any serum to help it. Care should be taken to avoid the entrance of other bacteria, as otherwise there may be some destruction of phagocytes or of bacteria in the incubator. Also unsterilised bacteria of more than one kind may overwork the phagocytes, whose capacity is limited. Whenever a bacterial suspension may have to be kept for some time it is advisable to sterilise it. Tubercle bacilli are agglutinated by the serum of a tuberculous patient, which interferes with the accuracy of the test. This agglutination can be prevented by heating the bacilli to $100^{\circ} \mathrm{C}$. A bacterial suspension which is too thick or too thin or unevenly mixed causes misleading results. The most convenient strength is such as will give an average of three or four bacilli per phagocyte, but any number between one and ten will suffice. Where the numbers are low an extra number of phagocytes should be inspected - say, 100 or more, instead of 50 per slide. If decided clumps of bacteria are seen in the slides they should be rejected. Such a slide is unreliable and gives too low an index. In performing this test the blood corpuscles, suspension, and serum should be taken in this order, as otherwise the corpuscles or suspension may be contaminated. The most serious error of this kind is where a particular serum contaminates the other tubes. Contamination of the bacillary suspension with blood corpuscles is less objectionable than the reverse. Care should be taken to draw the suspension from the upper part of the tube and to prepare more than is actually necessary, as the lowest layers will be perceptibly thicker than the upper. In mixing the three constituents some of the phagocytes may be left behind if they are drawn too rapidly up and down the pipette. Accuracy in measuring the quantities is, of course, necessary, but with reasonable care and skill the error need not be great in this respect.

With imperfect appliances it may happen that the incubator is not kept at the right temperature. One cannot expect to obtain satisfactory results with a variable tempera. ture, or one which is too high or too low. Also, I think it advisable to keep the pipettes horizontal in the incubator, to prevent gravitation and unequal distribution. In making the slide preparation it is essential to spread the material in a very thin film, with a definite edge well within the margin of the glass, to avoid difficulties in counting. I have found it convenient to spread in a series of jerks, making a wavy or striated film; but Wright is in the habit of making the film with one continuous sweeping movement. For some time past I have been using for a spreader a piece of glass divided lengthwise, as recommended by Allen, as it gives two edges to the film each of which can be easily examined. If the film is too thick the phagocytes remain rounded instead of being flattened out, in which case bacilli may escape notice along the edges of the phagocyte and elsewhere. Too deep a staining of the phagocyte or insufficient staining of the bacilli may also lead to errors. On the other hand, if the phagocytes are too faintly coloured they may be altogether missed. Films are easily spoilt by insufficient decolouration with acid, but such a mistake would not be made by anyone who has had sufficient practice. Wright is of opinion that the Ziehl-Neelsen method of staining destroys some of the phagocytes, and has therefore abandoned it for the aniline or fuchsin method. I am, however, under the impression that the degree of heat used with the Ziehl-Neelsen solution has something to do with the result. If the hot stain is poured over the slides in a cool photographic dish, the specimens can be well stained without any damage. This method may not be quite so expeditious as Wright's own method, but several dishfuls of slides ean be fixed and stained by it in less than an hour. In counting the bacilli, the same rnles should be observed for all the specimens alike. Slides with clumps of bacilli or large masses of cells should be rejected. Fragments of bacilli should either be counted as distinct bacilli or as fractions of a bacillus. Uncountable clusters of bacill should not exist in a properly prepared specimen; if a few are present, the numbers should be estimated as nearly as possible. It is customary in some laboratories to put down each such cluster as ten bacilli. All bacilli touching a phagocyte should be included in the count.

In examining a slide it should be remembered that phagocytes vary greatly in apparent size, according to the extent to which they have been flattened out. Mononuclears, even if covered with bacilli, are best disregarded. Owing to the arrangements at St. Mary's Hospital it has been possible to do much of the counting in duplicate or by three people in succession; and judged by this check, Wright is of opinion that with reasonable care there should not be a greater error than 10 per cent. Fleming (loc, cit.) gives a number of comparative figures which substantiate this statement. From time to time I have had the opportunity of comparing my own work with that of other laboratories, and have found the difference in results very small.

Clinical applications of the opsonic test must be left for another communication.

Farnham.

\section{A CASE OF MULTIPLE ANEURYSMS OF THE AORTIC ARCH AND THORACIC AORTA.}

BY H. EMLYN JONES, M.R.C.S. ENG., L.R.C.P. LoND., LATE HOUSE PHXSICTAN, LONDON HOSPITAL;

AND

T. H. C. BENIANS, M.R.C.S. ENG., L.R.C.P. LOND., ASSISTANT TO THE PATHOLOGICAL INSTITUTE, LONDON HOSPITAL.

THE patient, a man, aged 45 years, a shopkeeper, was admitted into the London Hospital, under the care of Dr. Cecil Wall, on June 27th, 1908, complaining of a swelling in the upper part of the left side of the chest, together with radiating pain over the left shoulder, shooting down the left arm, causing numbness of the fingers, and hæmoptysis. The illness commenced two and a quarter years previously, when he seemed to have " cold upon cold," and finally, two years ago, suddenly brought up two to three pints of blood. $\mathrm{He}$ attended the hospital out-patient department, and was diagnosed at that time as "Old hæmoptysis. Consolidation of the right apex (? tubercle). Neuritis of the right cervical plexus." The patient described this pain as extremely acute, often causing him to keep his head bent towards the right side. $\mathrm{He}$ also had shooting pains along the right arm, similar to those he described on admission in June, 1908, as occurring along the left arm. The symptoms abated somewhat and he remained fairly well until October, 1907, when he commenced to experience the pain above described in the regions of the left shoulder and left arm.

On admission on June 27th, 1908, he complained of : 1. A pulsating swelling over the inner end of the left clavicle of about the size of a small hen's egg. This swelling was first noticed three months previously. It was very painful and tender and rapidly increasing in size. 2. Slight cough and shortness of breath. 3. A coldness and numbness of the extremities, especially the hands and feet, which he could not keep warm. 4. Hæmoptysis; small clots occasionally; no further large quantity was expectorated after the hæmoptysis at the commencement of the illness. 5. Inability to use his right eye. This was found to have been present since birth and was on account of a "congenital ptosis." The tumour had expansile pulsation, was dull on light percussion, and gave a sense of fluctuation. The skin over it was slightly reddened. The area of cardiac dulness was slightly increased, but resonance was present between this and the dulness over the tumour. The heart sounds were normal. Anæmia was marked and the superficial veins of the upper part of the thorax were slightly dilated. The pressure of the carotid pulse was 108 . No pulsation was palpable at either wrist; the right temporal was palpable, but not the left.' The carotid pulses were markedly únequal and asynchronous. No tracheal tugging was present, nor was there any laryngeal paralysis. The voice was somewhat hoarse, but the cough was not 
"brassy." No glands were felt. The hands and feet were quite cold. There were crepitations present at both apices, whilst at the right apex the breath sounds were bronchial in character and at one spot whispering pectoriloquy was present. There were proptosis and divergence of the right eye, but the eye movements were free. The pupils were small but unequal and reacted both to light and on accommodation. The fundi and media were normal. There was no history or sign of past syphilis. The patient was extremely temperate, and had never done any really hard manual labour. The temperature was raised to $100^{\circ} \mathrm{F}$. at night. The urine was natural. Skiagrams taken on admission showed three distinct bulgings of the thoracic aorta: (1) of the descending aorta; (2) at the junction of the transverse and descending arch; and (3) of the ascending arch near the spot where the right innominate artery takes its origin.

Subsequent history. - The tumour on the left side gradually got larger until it reached the size shown in the accompanying illustration taken a few days before death. No

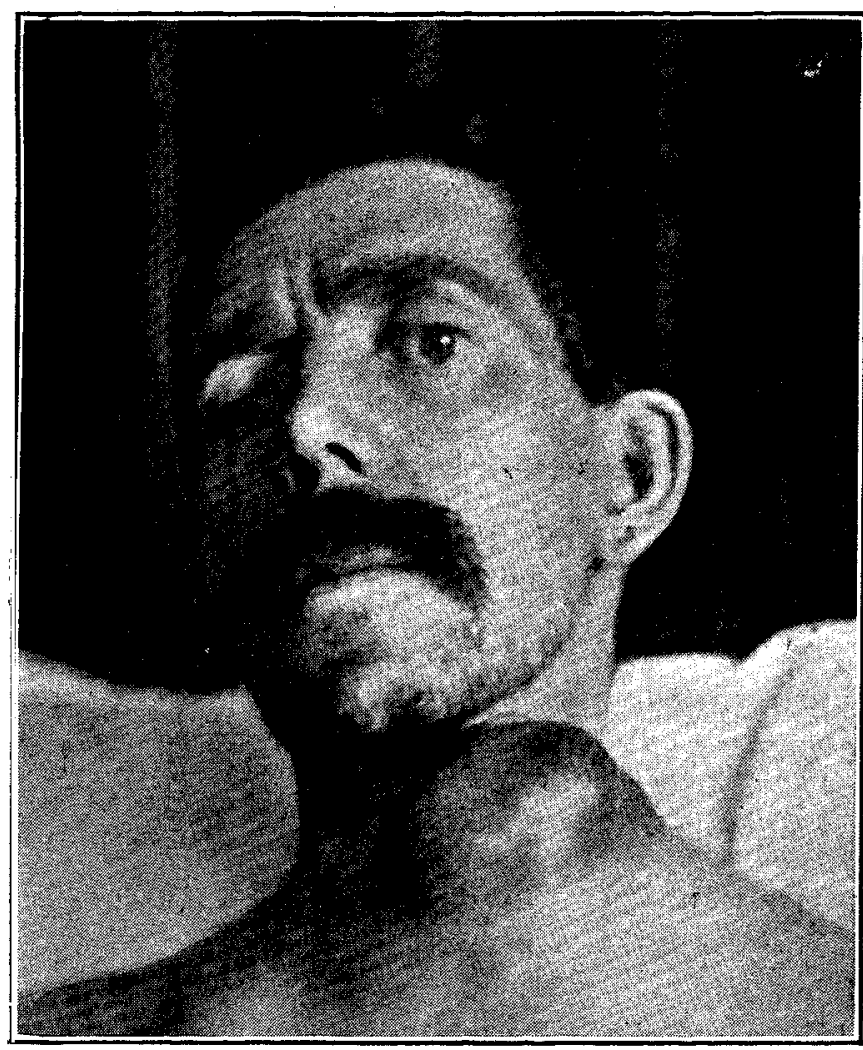

Reproduction of photograph showing the two dilatations of the arch present just before death. The large one on the left ruptured. The illustration also shows the ptosis present from birth on the right side.

murmur was ever heard over it. It remained an expansile pulsating tumour, the skin over it becoming more and more red until, on Sept. 10th, it gave way and general oozing from the sac commenced. This partially stopped for a few days, but a week later the large clot which had formed gave way and the patient immediately died.

About four weeks previously another tumour gradually made its appearance on the right side in the region of the right sterno-clavicular articulation, evidently the old dilatation at the junction of the ascending and transverse arches again becoming patent and approaching the surface. This tumour also had expansile pulsation, but no murmur was heard. The radial and temporal pulses remained absent and the physical signs at the apices of the lungs practically the same until death.

Summary of necropsy.-Extreme anæmia. Rupture of large aneurysm of the arch and ascending portion of the thoracic aorta through an erosion of the skin between the first and second ribs on the left side. Dislocation upwards of the left clavicle and separation of the first rib from its cartilage. Erosion of the periosteum of the clavicle, first rib, and adjacent sternum on the left side. Thrombosis of the right subclavian, left common carotid and subclavian arteries at their origins. Small erosion of the trachea. In communication with the aortic aneurysm a saccular aneurysm of the innominate artery protruding through the first intercostal space on the right side beneath the pectoralis major. Erosion of periosteum of first and second ribs and adjacent sternum on the right side. Slight dilatation and mesaortitic scarring in the upper portion of the descending thoracic aorta; button atheroma of the lower part of the descending thoracic aorta and atherema of the aortic commissure; calcareous plaque in intima of abdominal aorta close to bifurcation. Post-mortem staining of aorta. Calcareous
atheroma of coronary arteries; cerebral arteries free. Great $\propto$ dema of both arms. Small patches of fibrocaseous tubercle behind and below the apex of the left lung and in the apex of the left lower lobe. Fibrocaseous nodule in the course of a bronchus behind and below the apex of the right lung. Anthracosis and odema of lungs. Obliterating fibrous pleurisy right; fibrous pleural adhesions over leit apex. Anthracosis of tracheal and bronchial glands. Small fibrous scars on surface and within substance of liver ( 3 pounds $13 \frac{1}{2}$ ounces). Submiliary tubercles in liver. Miliary tubercles in cortex of both kidneys (together 14 ounces). Soft spleen, with hypertrophy of Malpighian bodies ( $5 \frac{1}{2}$ ounces). Calcareous mesenteric glands. Slight fibrosis of right testicle. Small polyp in splenic flexure. Mucous catarrh of stomach. No scars on legs or penis.

To the right of the sternum was a slight swelling between the second rib and the inner end of the clavicle. Immediately to the left of the sternum, over the first rib, there was a ragged ulcer of the skin ; this was approximately circular, about two inches in diameter, and filled with red gelatinous clot. When this clot was removed the base of the ulcer was seen to be formed by a mass of firm yellow laminated clot in which lay the left border of the sternum, the first rib, and the clavicle. These bones were devoid of periosteum and eroded. Further, the first rib was separated from its junction to the costal cartilage, and the clavicle was separated from its sternal articulation and lifted half an inch upwards and forwards. When the skin and subcutaneous tissues had been reflected the swelling on the right side was found to be caused by a mass of soft, granular, yellowish clot, which had broken through the pectoral muscles and extended inwards on to the anterior surface of the sternum. This clot protruded through an aperture in the first intercostal space on the right side close to the sternum, and the sternum and first and second ribs in this region were devoid of periosteum and eroded.

The removal of the sternum and the costal cartilages of the third to the ninth ribs exposed a large saccular dilatation of the aorta, which, at and above the level of the second ribs, filled the thoracic cavity almost completely and was firmly adherent to the chest wall. The dilatation was approximately of the shape of a heart, the apex of the "heart" lying at the commencement of the aorta. The greatest, aiameters of this sac were: vertically, 4 inches; anteroposteriorly, 3 inches; and from side to side, 5 inches. The upper border showed a mesial and two lateral convexities. The right lateral convexity was continuous with the mass of clot which had burst through the first intercostal space on the right side. From its posterior aspect arose the right subclavian and common carotid arteries, so that this portion of the aneurysm was formed, in part at any rate, by dilatation of the innominate artery. The mesial convexity was continuous with the clot which had ulcerated the skin to the left of the sternum. From the posterior aspect of this arose the left common carotid and subclavian arteries.

On opening the aorta the semilunar valves were found to be competent to the water test; the commissure and sinuses of Valsalva were not dilated. The intima of the lower inch of the ascending aorta was stained a deep red and showed large irregular yellow thickenings. There was no definite scarring. The dilatation of the aorta commenced abruptly one inch above the commissure. On the posterior wall of the dilated cavity was a Y-shaped ridge which divided the cavity into a superomesial and two lateral sacs, corresponding to the three convexities seen externally.' The interior of the right sac, which corresponded to the dilated innominate artery, was covered by a layer nearly one inch thick of adherent, white, laminated clot. This clot. was found to be continuous through a large aperture in the wall with the soft clot which lay beneath the skin to the light of the sternum. The superomesial sac was also lined by firm laminated clot which completely covered the apertures of the left subclavian and common carotid arteries. In the anterior and upper part of the sac there was a deficiency in its wall through which the clot passed to form the base of the ulcer on the skin to the left of the sternum. When the clot was removed from its lowermost part, in the fork of the Y-shaped ridge, an aperture of the size of a pin's head was seen which opened into the trachea $1 \frac{1}{2}$ inches above its bifurcation. The left lateral sac lay. somewhat posteriorly to the other two ; in its lower and posterior part was an aperture leading into the descending aorta. This aperture was only $1 \frac{1}{2}$ inches from the point where the ascending aorta commenced to dilate; it had rounded margins and corresponded in size to the lumen of 
the slightly dilated descending thoracic aorta. On removal of the clot the wall of the aneurysm was found to be thin. The intimal surface was smooth and showed numerous small pits.

The upper two inches of the descending thoracic aorta were slightly dilated and the intima showed slightly raised thickenings of a yellowish-white colour, cartilaginous in appearance. Between these thickenings the intima showed longitudinal wrinkles and some deep pits. On section the media was narrow and ill-defined, whilst the thickened intima was fibrotic. In the lower part of the descending thoracic aorta and in the abdominal aorta there were numerous large nodular thickenings, between which the intima showed some fine longitudinal wrinkles. These nodules were seen on section to be formed by a thickening of the intima, which was firm and cartilaginous in its upper part, but soft and yellow in its deeper layers. The media was well defined and only slightly, if at all, narrowed below the intimal thickenings. Close to the bifurcation of the aorta was a smooth calcareous plaque in the intima.

Of the four large arterial trunks arising from the aneurysm the right common carotid was the only one the opening of which was not completely occluded by the firm clot lining the sac ; the opening of this artery was wide. The lumen of the right common carotid was somewhat dilated. The lumen of the left common carotid contained no clot, but was considerably contracted and in its lowest $1 \frac{1}{2}$ inches was barely patent. The subclavian arteries were of normal calibre.

Microscopic examination. - The aorta. Eight portions were removed for microscopic examination from the aorta on the distal side of the aneurysm. Two of these were sections of the nodules in the lower part of the descending thoracic aorta and in the abdominal aorta; they showed under the microscope the changes characteristic of atheroma. The intima was thickened and formed nodular elevations. At either side of the nodules two distinct layers could be distinguished in the thickened intima, an inner fibrotic layer, and an outer layer which contained stout elastic fibres and corresponded to the hypertrophic elastic and the longitudinal muscle elastic layers of Jores. In the nodular elevations the outer of these two layers was, with the exception of its innermost portion, entirely replaced by structureless débris, in which there was some calcium. The media was unaltered or a few of its innermost elastic fibres were destroyed. The adventitia showed no abnormality. The remaining portions were removed from the upper part of the thoracic aorta where the intima was wrinkled and pitted. They showed ander the microscope the appearances of mesaortitis. The intima was thickened and consisted of stout connective-tissue fibres with very little elastic. It was seldom possible to distinguish individual layers in the thickened intima, and there was no degeneration of the connective-tissue fibres. The media was very narrow. In places it was replaced by fibrous tissue which united the intima to the adventitia; in others it contained patches of fibrous tissue, whilst its elastic fibres were swollen, tortuous, and darkly stained, and were arranged in small isolated groups. Vessels passed from the adventitia through the media to the fibrotic intima; these vessels were surrounded by a zone of round-cell infiltration, which in a few places was of considerable breadth. The adventitia was thick and fibrotic and some of the arteries showed an endarteritis fibrotica.

The liver.-Large fat droplets were seen in the cells of the periphery of most of the lobules in sections stained with Sudan. Close to some of the portal systems were small rounded granulation areas which consist of a loose network of swollen spindle cells, with numerous lymphocytes in the periphery. The centres of these areas were often necrosed and in one was a multinuclear giant cell with no definite border and with the nuclei near its centre. There were also areas in which the parenchyma was occupied by a network of fibrotic strands, most of which were connected with portal systems. These strands were formed in their central parts by connective-tissue fibres with spindle nuclei, and between the fibres were some proliferating bile-ductules and a few isolated liver cells; in the periphery of the strands were numerous lymphocytes. One or two of the strands were continuous with the rounded granulation areas. Within the meshes formed by these fibrous strands the parenchyma consisted of columns of large cells. No tubercle bacilli and no spirochætæ could be detected in specially stained preparations.
The kidney. - In the cortex was a small granulomatous nodule. Its centre consisted of a structureless substance which was stained deeply by eosine and contained nuclear fragments ; round this was a zone of closely packed connective tissue cells with spindle nuclei, whilst in the extreme periphery were lymphocytes. Only one portion of the kidney was reserved for microscopic examination, and in this. no giant cells and no tubercle bacilli could be found in the granulomatous nodule.

Remarks.-The interesting features of this case are: 1. The absence of any of the usual determining circumstances of aortic aneurysm-viz., syphilis, alcohol, hard work, \&c., or of any evidence of a long-sustained high blood pressure. 2. The extraordinary tendency for clotting and attempts at spontaneous cure. Even when rupture took place and oozing commenced this stopped for a few days, a large clot forming, until the final more extensive rupture proved immediately fatal. 3. The old history of hæmoptysis, evidently one of the dilatations (probably that of the descending arch) ulcerating into the trachea two years previously. The ulceration on the trachea was seen post mortem, and though old tubercle was present at the left apex, no extensive tuberculous process had invaded the lung, making it practically certain that the large sudden hæmoptysis was of aneurysmal origin. 4. The spontaneous cure of the aneurysm of the right side with cessation of symptoms, followed two and a half years later by it again becoming patent after the appearance of the larger and more rapidly growing dilatation of the left side. 5. The multiplicity of the dilatations.

Our best thanks for permission to publish this case are due to Dr. Cecil Wall, under whose care the patient was, and to Dr. Hubert M. Turnbull, Director of the Pathological Institute.

\section{A REPORT UPON THE ROUTINE USE, BY THE OPEN METHOD, OF A MIXTURE OF CHLOROFORM AND ETHER. ${ }^{1}$}

BY FREDERIC W. HEWITT, M.V.O., M.A., M.D. CANTAB., ANESTHETIST TO HIS MAJESTY THE KING; PHYSTCIAN-AN-FSTHETIST TO ST. GEORGE'S HOSPITAL; CONSULTING AN ESTHETIST AND EMERITUS LECTURER ON ANASTHETICS AT THE LONDON HOSPITAI, ETC.

AND

JOSEPH BLUMFELD, B.A., M.D., B.C. CANTAB., SENIOR AN ESTHETIST TO ST. GEORGE'S HOSPITAL, ETC.

1. The anesthetic. - The mixture is made of chloroform 8 parts by volume and ether 3 parts by volume. In hospital cases Duncan and Flockhart's methylated (red label) chloroform and Huskisson's "pure methylated" ether were used to make the mixture. A similar mixture was usually employed in private practice, though in certain cases one with ethyl-alcohol constituents was used for purposes of comparison.

2. The method of use and apparatus employed. - The mask employed (Fig. 1) is essentially a Skinner's mask and consists of a wire frame over which is stretched a single layer of thin flannel. The mask thus formed presents an oval opening 5 inches by 3 inches and is capable of close adaptation to the face. The vault of the mask is high enough not to touch the nose when its rim rests upon the face. The size of the mask and the material with which it is covered are important details. It is found, for instance, that if two layers of the thin flannel are used instead of one an equal quantity of anæsthetic produces different effects in the two cases. The small tube passing through the middle of the stretched flannel is only of use when oxygen is required. A mask of the same dimensions, but with $a$ guttered rim, a handle, and no oxygen tube, has generally been used by one of us (J. B.). In using the mixture a gradual process is followed. The mask is applied to the face and, after a few breaths, the mixture is added, a few drops at a time. A regulating drop bottle is used capable of delivering the mixture in isolated drops, in a rapid series of drops, or in a continuous stream. (Fig. 2.) During the first two minutes the mixture is poured upon the mask in such small quantities at a time that there is never more than a quarter

1 To be presented to the Anæsthetics Committee of the British Association for the Advancement of Science, August, 1909. 